DE

M E D I C I N A

T R O P I C A L

$\mathrm{DE}$

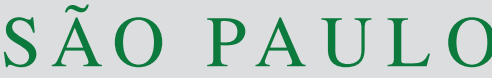

JOURNAL OF THE SÃO PAULO INSTITUTE OF TROPICAL MEDICINE

1Universidade de São Paulo, Instituto de Medicina Tropical de São Paulo, São Paulo, São Paulo, Brazil

2Universidade de São Paulo, Faculdade de Medicina, Hospital das Clínicas, Laboratório de Investigação Médica (LIM 06), São Paulo, São Paulo, Brazil

${ }^{3}$ Universidade Federal de Ouro Preto, Departamento de Ciências Biológicas, Ouro Preto, Minas Gerais, Brazil

${ }^{4}$ Universidade Federal de Uberlândia, Laboratório de Diagnóstico de Parasitoses, Uberlândia, Minas Gerais, Brazil

Correspondence to: Fabiana Martins de Paula

Universidade de São Paulo, Faculdade de Medicina, Hospital das Clínicas, Laboratório de Investigação Médica (LIM 06),, Av. Dr. Enéas de Carvalho Aguiar, 470, CEP 05403-000, São Paulo, SP, Brazil

E-mail: fabiana.paula@hc.fm.usp.br

Received: 24 October 2019

Accepted: 30 March 2020

\section{Immunoreactivity of proteins within 30-40 kDa range during the acute and the recovery phases in rats experimentally infected with Strongyloides venezuelensis}

\author{
Priscilla Duarte Marques Fonseca ${ }^{1}$, Marcelo Andreeta Corral ${ }^{2}$, Dirce \\ Mary C. Lima Meisel ${ }^{2}$, Debora Levi ${ }^{2}$, Rafael Correa Nascimento', William \\ Castro-Borges $^{3}$, Ronaldo Gryschek ${ }^{1,2}$, Julia Maria Costa-Cruz ${ }^{4}$, Fabiana \\ Martins de Paula ${ }^{1,2}$
}

KEYWORDS: Strongyloides venezuelensis. Acute and recovery phases. 30-40 kDa, IgG.

Strongyloides venezuelensis has been used in rodent models of Strongyloides infection, particularly in immunological studies ${ }^{1}$ related to the production of heterologous antigens in the serological diagnosis of human strongyloidiasis ${ }^{2}$. In experimental infections, it is possible to define the acute and the recovery phases ${ }^{3}$, unlike human infections caused by Strongyloides stercoralis. In this context, antibody production and recognition of immunogenic bands during the acute and the recovery phases have not been well explored. Thus, the objective of this study was to evaluate the production of anti-Strongyloides $\mathrm{IgG}$ antibodies and the recognition of immunogenic bands produced during the acute and the recovery phases in rats experimentally infected with $S$. venezuelensis.

Male Wistar rats (Rattus norvegicus), with four weeks old, were obtained from the Bioterio de Producao de Ratos, Instituto de Ciencias Biomedicas, Universidade de Sao Paulo, Brazil and were kept in the Bioterio do Instituto de Medicina Tropical de Sao Paulo (IMT-SP). Rats received sterilized food and water ad libitum and were handled in compliance with the animal ethics guidelines adopted by the Comite de Etica em Experimentacao Animal, IMT (CEUA IMT 317A).

S. venezuelensis infective larvae (iL3) were obtained by charcoal culture of infected rats faeces (CEUA protocol IMT 0356A). The experimental infections were 
established in 35 rats divided into three groups: infected subcutaneously with 400 S. venezuelensis iL3 ( $\mathrm{n}=15$, 400iL3), infected with 4,000 S. venezuelensis iL3 ( $\mathrm{n}=15$, 4000iL3) and uninfected rats ( $\mathrm{n}=5$, negative control, NC).

The number of eggs per gram of faeces (EPG) was obtained daily until day 35 post infection (pi), according to the Gordon and Whitlock method ${ }^{4}$. EPG was performed in 5 samples of 1 gram of faeces randomly collected on each day post infection in each infected group (400iL3 and 4000iL3). The results were determined after five counts (mean \pm standard error). Blood samples (five animals) were collected by cardiac puncture on days 2,7 and 35 pi after anaesthesia with ketamine/xylazine, and the animals were subsequently euthanized. Blood samples were centrifuged and the serum samples obtained were used in ELISA and Western blotting.

Two antigenic fractions were prepared using approximately 200,000 S. venezuelensis iL3. Briefly, iL3 were resuspended in $1 \mathrm{~mL}$ of Tris- $\mathrm{HCl}(25 \mathrm{mM}$ [pH 7.5]) containing protease inhibitors (Sigma-Aldrich, St. Louis, MO, USA) and sonicated on ice (5 cycles of $20 \mathrm{~s}$ ). The suspensions were centrifuged at $12,400 \times g$ for $30 \mathrm{~min}$ at $4{ }^{\circ} \mathrm{C}$, and the supernatant was collected (soluble fraction, $\mathrm{SAg}$ ). Pellets were resuspended in $5 \mathrm{M}$ urea, $2 \mathrm{M}$ thiourea and 4\% 3-[(3-cholamidopropyl)dimethylammonio]-1propanesulfonate (CHAPS) in an ice bath for $30 \mathrm{~min}$, and the supernatant was collected after centrifugation at $12,400 \times g$ for $30 \mathrm{~min}$ at $4{ }^{\circ} \mathrm{C}$ (membrane fraction, $\mathrm{MAg}$ ).

ELISA was performed as described previously ${ }^{5}$, with some modifications. Microplates were coated overnight at $4{ }^{\circ} \mathrm{C}$ with $10 \mu \mathrm{g} / \mathrm{mL}$ (to a final volume $50 \mu \mathrm{L} /$ well) of each $S$. venezuelensis antigenic fraction in $0.06 \mathrm{M}$ carbonatebicarbonate buffer $(\mathrm{pH}$ 9.6). Plates were incubated with serum samples (1:20) for $45 \mathrm{~min}$ at $37^{\circ} \mathrm{C}$ and then with the secondary antibody consisting of peroxidase-labelled goat anti-rat $\operatorname{IgG}$ (Sigma-Aldrich) at a dilution of 1:2,000 for $45 \mathrm{~min}$ at $37^{\circ} \mathrm{C}$. The assay was developed by adding TMB chromogen solution (Invitrogen, Thermo Fisher Scientific, Waltham, MA, USA) for 15 min and was stopped by addition of $2 \mathrm{NH}_{2} \mathrm{SO}_{4}$. The optical density (OD) was determined at $450 \mathrm{~nm}$ in a plate reader (Thermo Fisher Scientific). Statistical analyses were performed using the GraphPad Prism software version 8.0 (GraphPad Software. San Diego, CA, USA). Statistical significance was determined by ANOVA, followed by Tukey's multiple comparison test $(p<0.05)$.

Electrophoresis and Western blotting were performed as previously described ${ }^{6}$. Briefly, approximately $140 \mu \mathrm{g}$ $(2 \mu \mathrm{g} / \mathrm{mm}$ of gel) of the antigenic fractions (SAg and MAg) underwent electrophoresis in $12 \%$ polyacrylamide gel (SDS-PAGE) for $2 \mathrm{~h}(20 \mathrm{~mA})$. A molecular mass standard (10-260 kDa; Bio-Rad Laboratories, Hercules, CA, USA) was used to quantitate the relative protein bands. After electrophoresis, the proteins on the gel were transferred to a polyvinylidene difluoride (PVDF) membrane $(0.2 \mu \mathrm{m})$ (Bio-Rad Laboratories). In the Western blotting, after blocking (50 mM Tris- $\mathrm{HCl}$ [pH 7.5]; $3 \%$ Tween 20, and 3\% milk), the membranes were incubated with sera diluted 1:50 in T buffer ( $50 \mathrm{mM}$ Tris- $\mathrm{HCl}$ [pH 7.5], $100 \mathrm{mM} \mathrm{NaCl}, 0.1 \%$ Tween 20 and 5\% milk). The secondary antibody (anti-rat IgG conjugated with peroxidase; Sigma-Aldrich) was then diluted 1:2,000 in $\mathrm{T}$ buffer and added to the membrane. Binding was detected using ECL Prime Western Blotting detection reagents (GE Healthcare Life Sciences, Little Chalfont, UK). The antigenic components were visualized in a Luminescent Image Analyzer (Fujifilm, Minato, Tokyo, Japan) and analysed by the VisionWorks LS Analysis Software (Analytik Jena, Jena, Germany).

The egg count showed the development of the experimental infection (Figure 1A). The acute and the recovery phases in the experimental infection with $S$. venezuelensis were well characterized by Chiuso-Minicucci et al. ${ }^{3}$ The acute phase was identified by elimination of a large number of eggs in the faeces on days 6-14 pi (Figure 1A). On day $8 \mathrm{pi}$, the egg excretion peaked with $14,600 \pm 1,702.9$ and $43,870 \pm 801.5 \mathrm{EPG}$ in the rats infected with $400 \mathrm{iL} 3$ and $4000 i L 3$, respectively. The recovery phase was defined by a reduction in egg excretion and resolution of infection between days 30 to 35 pi (Figure 1A). The increase in the EPG on day 8 pi and the reduction after day 13 pi have been reported in the literature in rats experimentally infected with S. venezuelensis ${ }^{3,5,7}$. Although infection with $S$. venezuelensis is well tolerated by rats, ${ }^{1}$ infections with $400 \mathrm{iL} 3$ and $4000 \mathrm{iL} 3$ can be considered moderate and severe, respectively ${ }^{8,9}$.

The immune response during the acute and the recovery phases in experimental infection with $S$. venezuelensis was shown by Chiuso-Minicucci et al. ${ }^{3}$ In this study, the specific $\mathrm{IgG}$ for $S$. venezuelensis was lower in the acute phase than in the recovery phase. Our results showed differences in IgG levels in the acute and the recovery phases (Figure 1B). There was a considerable increase in the OD values for the SAg during the experimental infection, suggesting the differentiation of the acute and the recovery phases, independent of the inoculum. Increased production of antibodies in the recovery phase in rats infected with 2000 iL3 has been observed previously ${ }^{5}$. On the other hand, previous studies have shown higher OD values on day 8 pi compared with other days pi in animals infected with $S$. venezuelensis ${ }^{7}$ and $S$. ratti ${ }^{10}$.

Comparing the infections with 400iL3 and 4000iL3, a statistical difference $(p<0.05)$ was observed for OD values only on day 7 pi, considering the two antigens (Figure 1B). The results showed a significant increase in OD values 
(A)

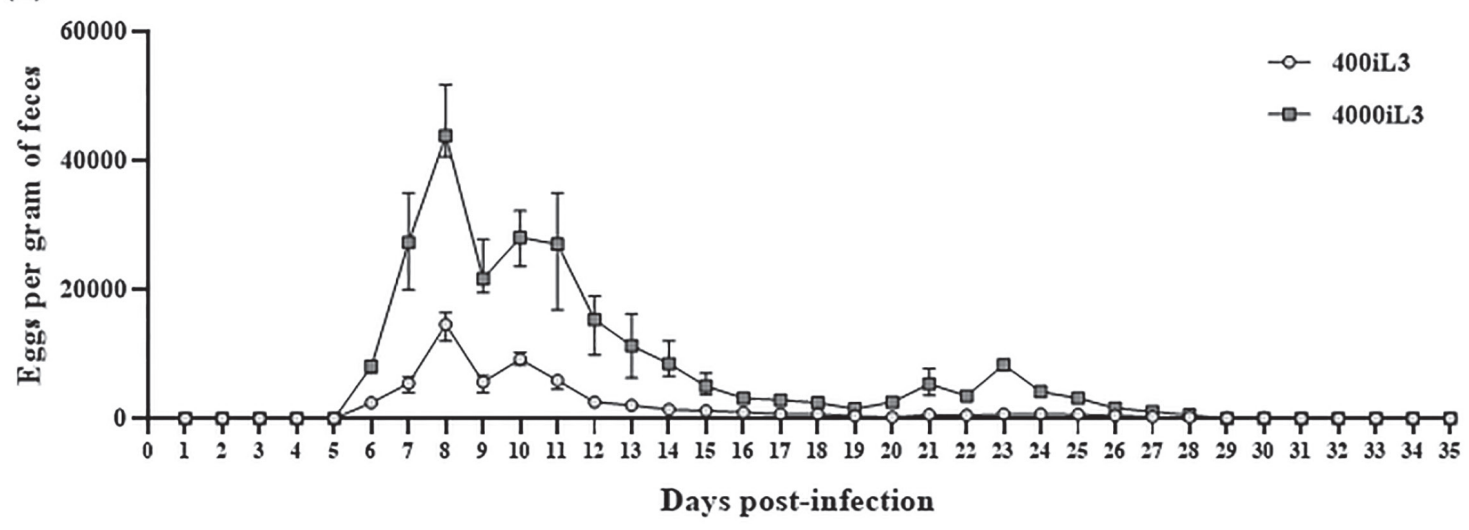

(B)

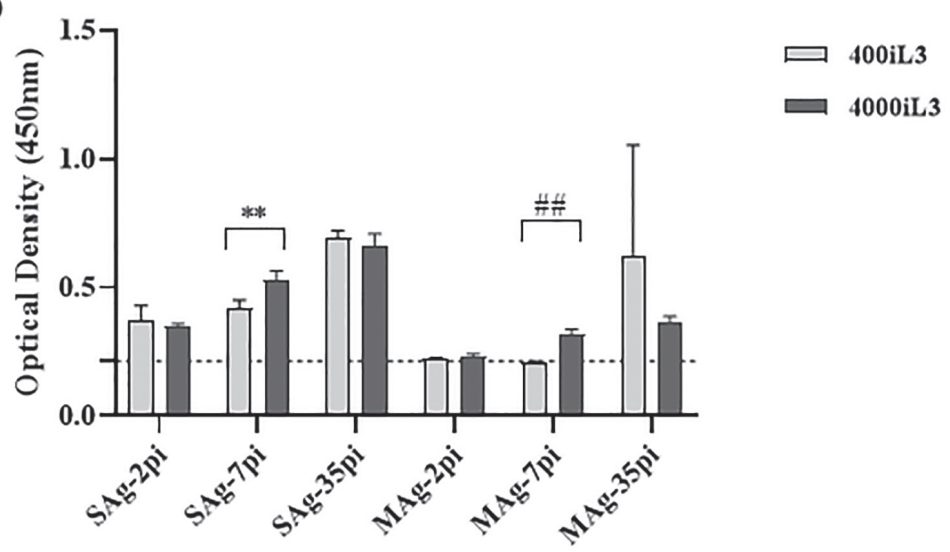

Figure 1 - Eggs per gram of faeces (A) and IgG anti-S. venezuelensis in serum samples (B) from rats experimentally infected with 400iL3 and 4000iL3 S. venezuelensis. (A) Results are expressed as means \pm SEM of five rats per group. (B) ELISA test using soluble (SAg) and membrane (MAg) antigens from $S$. venezuelensis filariform larvae on days 2, 7 and 35 post infection (pi). Data are expressed as means $\pm \operatorname{SEM}(\mathrm{n}=5),{ }^{\star \star} p=0.0055$, \#\# $p=0.0024$. Dashed lines indicate the mean optical density of the negative control $\pm \operatorname{SEM}(n=5)$.

in rats infected with 400iL3 on day 2 pi versus day 7 pi independent of antigens; and on day 35 pi versus 2 pi and 7 pi, only the SAg showed a difference. In addition, for rats infected with $4000 \mathrm{iL} 3$, there were statistically significant differences on days 2, 7 and 35 pi for the two antigens and on day 2 pi versus 7 pi for each antigen. Analysing the OD values, we can observe that the 4000iL3 group showed lower values in relation to $400 \mathrm{iL} 3$ by MAg. These results could be related to variations in the group, because S. venezuelensis infection is well tolerated by rats ${ }^{1}$.

Few studies have evaluated immunogenic bands during experimental infection ${ }^{10-12}$. In the present study, immunogenic bands were recognized (Figure 2), independent of the antigenic fraction and the phase of infection. Bands of $\sim 100-140 \mathrm{kDa}$ were recognized in two groups independently, in the infection phase by SAg and in the recovery phase by MAg. We observed the $\sim 65-70 \mathrm{kDa}$ bands in the recovery phase in the 400iL3 and 4000iL3 groups, mainly using SAg. In addition, there was greater staining intensity of protein bands visualized in MAg. In a recent study of experimental strongyloidiasis ${ }^{11}$, immunoreactive bands at 17,38 and $50 \mathrm{kDa}$ were demonstrated using $S$. venezuelensis iL3 alkaline extract. In addition, 36, 68, 76, 83 and $102 \mathrm{kDa}$ bands for a saline extract were considered antigenic in another experimental investigation with $S$. venezuelensis ${ }^{12}$.

The 30-40 kDa band was recognized in the acute and recovery phases of infection in both groups. In agreement with previous reports ${ }^{10-12}$, this mass range has been considered an immunogenic band in experimental strongyloidiasis. In addition, 30-40 kDa bands are considered important for the diagnosis of $S$. stercoralis infections $s^{6,13,14}$. Recognition of this protein range by $\operatorname{IgG}$ antibodies from the serum of rats infected with 400iL3 may reinforce its potential diagnostic value. In addition, it is difficult to define the phases in S. stercoralis infection, which makes recognition of the 30-40 $\mathrm{kDa}$ band, mainly by MAg, an important diagnostic tool.

Considering the difference in protein extracts and the intrinsic variability of the reaction, it can be suggested that the OD values and the recognition of immunogenic bands 
(A) $\mathrm{SAg}$

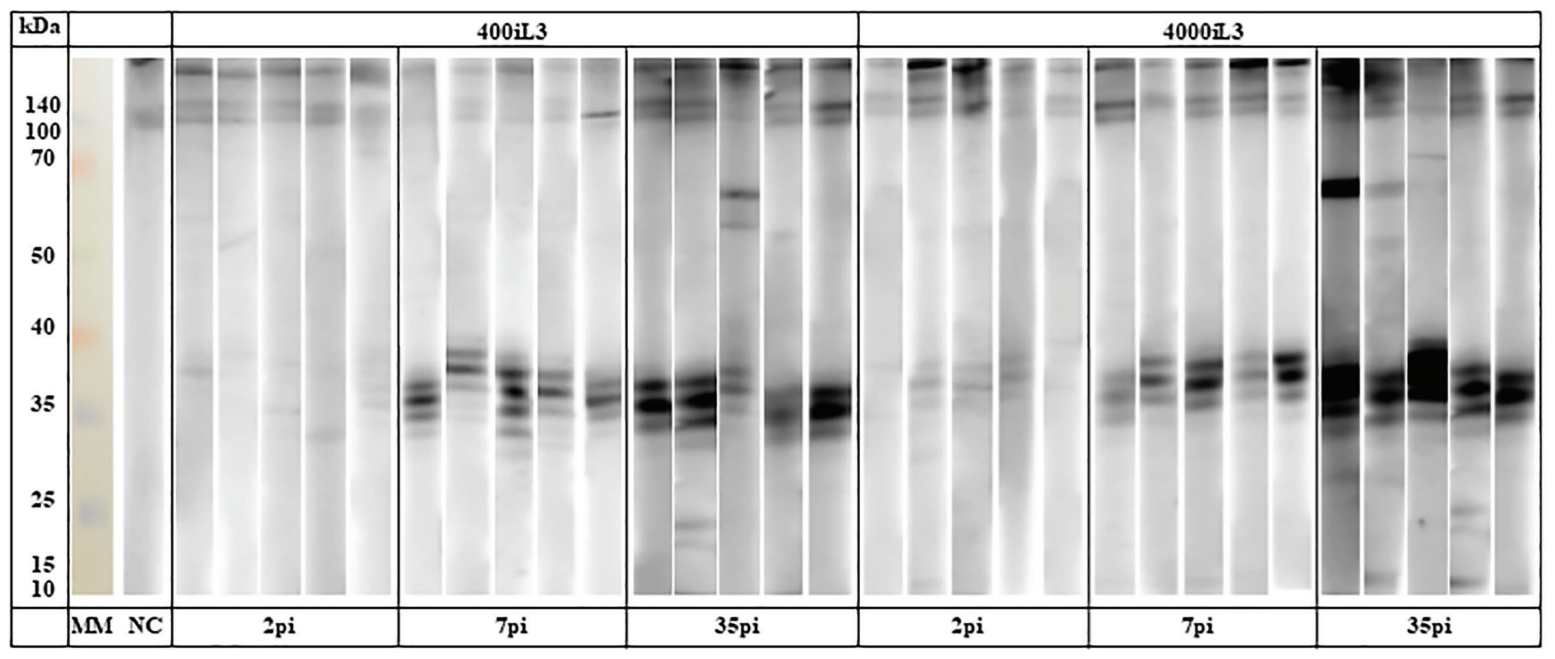

(B) MAg

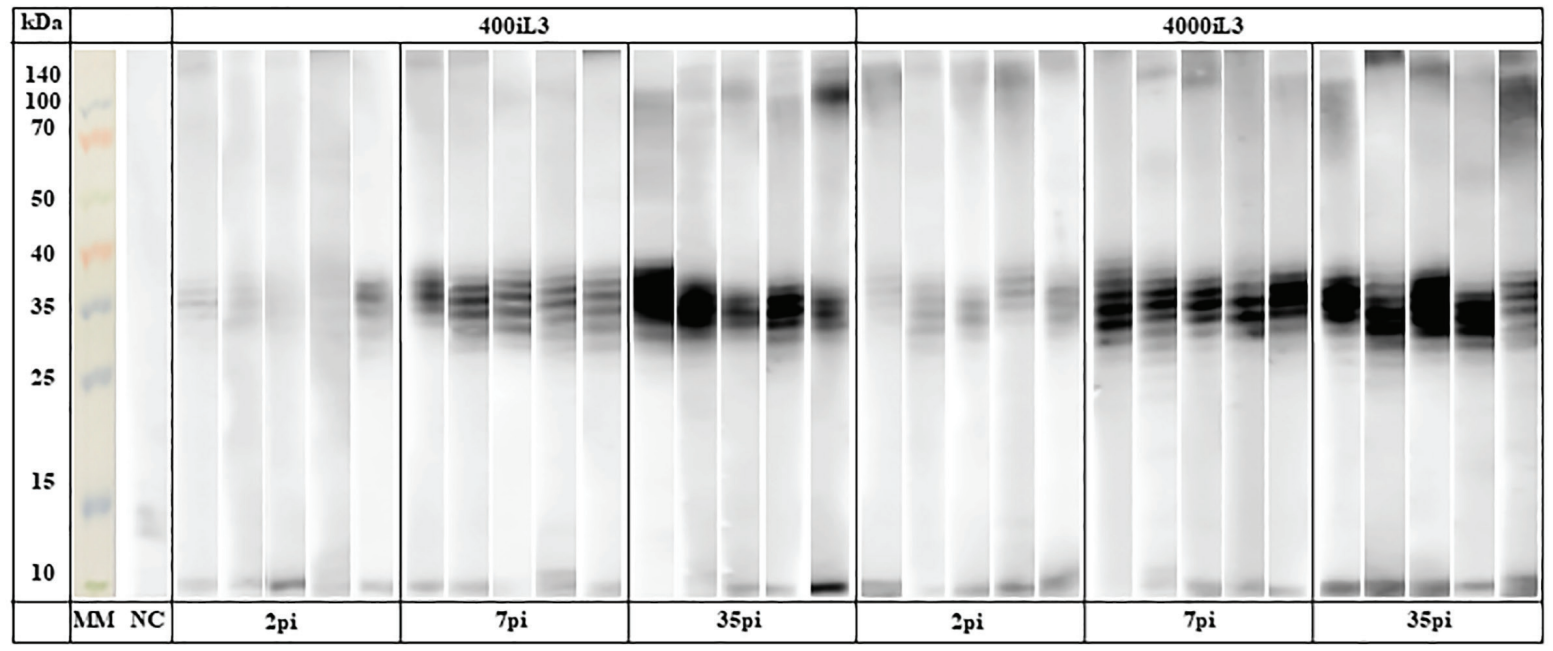

Figure 2 - Recognition of antigenic bands (soluble fraction [SAg, A] and membrane fraction [Mag, B]) in serum samples from rats experimentally infected with S. venezuelensis (400iL3 and 4000iL3) on days 2, 7 and 35 post infection (pi) $(n=5)$.

occurred according to the phases of the infection. Based on the literature ${ }^{15}$, the increase in OD values was related to the increase in the number of immunogenic bands recognized.

Therefore, experimental S. venezuelensis infection is an important tool for diagnostic evaluations for use in human strongyloidiasis. Thus, the present study points to the use of SAg in ELISA and highlights the proteins within the 30-40 $\mathrm{kDa}$ range in MAg as a tool for the diagnosis of the acute and the recovery phases in experimental infection by S. venezuelensis.

\section{CONFLICT OF INTERESTS}

None.

\section{FUNDING}

This research was supported by Fundação de Amparo à Pesquisa do Estado de São Paulo (grant
No FAPESP 2016/06185-0, to FMP), and Coordenação de Aperfeiçoamento Pessoal de Nível Superior (grant $\mathrm{N}^{\circ}$ CAPES 0001, to PDMF), Brazil.

\section{REFERENCES}

1. Viney M, Kikuchi T. Strongyloides ratti and S. venezuelensis: rodent models of Strongyloides infection. Parasitology. 2017;144:285-94.

2. Levenhagen MA, Costa-Cruz JM. Update on immunologic and molecular diagnosis of human strongyloidiasis. Acta Trop. 2014;135:33-43.

3. Chiuso-Minicucci F, Marra NM, Zornella-Pezavento SF, França TG, Ishikawa LL, Amarante MR, et al. Recovery from Strongyloides venezuelensis infection in Lewis rats is associated with a strong Th2 response. Parasite Immunol. 2010;32:74-8.

4. Gordon HM, Whitlock HV. New technique for counting nematode eggs in sheep faeces. J Counc Sci Ind Res. 1939;12:50-2. 
5. Marques PD, Malta FM, Meisel DM, Corral MA, Pinho JR, Costa-Cruz JM, et al. Diagnosis of the strongyloid nematode Strongyloides venezuelensis in experimentally infected rats. J Helminthol. 2016;90:422-7.

6. Corral MA, Paula FM, Meisel DM, Castilho VL, Gonçalves EM, Levy D, et al. Potential immunological markers for diagnosis of human strongyloidiasis using heterologous antigens. Parasitology. 2017;144:124-30.

7. Gonçalves AL, Silva CV, Ueta MT, Costa-Cruz JM. Antigen, antibody and immune complex detection in serum samples from rats experimentally infected with Strongyloides venezuelensis. Exp Parasitol. 2012;130:205-8.

8. Marra NM, Chiuso-Minicucci F, Machado GC, ZorzellaPezavento SF, França TG, Ishikawa LL, et al. Faecal examination and PCR to detect Strongyloides venezuelensis in experimentally infected Lewis rats. Mem Inst Oswaldo Cruz. 2010;105:57-61.

9. Nakai ES, Amarante AF. Infecção experimental de camundongos (Mus musculus) e ratos (Rattus norvegicus) com Strongyloides venezuelensis. Rev Bras Parasitol Vet. 2001;10:1-6.

10. Rodrigues RM, Cardoso CR, Gonçalves AL, Silva NM, Massa $\mathrm{V}$, Alves R, et al. Increased susceptibility to Strongyloides venezuelensis infection is related to the parasite load and absence of major histocompatibility complex (MHC) class II molecules. Exp Parasitol. 2013;135:580-6.
11. Goulart de Carvalho EF, Neto de Sousa JE, Gonçalves AL, CunhaJunior JP, Costa-Cruz JM. Immunoblotting using Strongyloides venezuelensis larvae, partenogenetic females or eggs extracts for the diagnosis of experimentally infected immunosuppressed rats. Exp Parasitol. 2015;157:117-23.

12. Sousa JE, Carvalho EF, Levenhagen MA, Faria LS, GonçalvesPires MR, Costa-Cruz JM. Serological cross-reactivity between Strongyloides venezuelensis and Shyphacia muris in Wistar rats (Rattus norvegicus). Parasitol Int. 2016;65:137-45.

13. Sudré AP, Siqueira RC, Barreto MG, Peralta RH, Macedo HW, Peralta JM. Identification of a $26-\mathrm{kD}$ a protein fraction as an important antigen for application in the immunodiagnosis of strongyloidiasis. Parasitol Res. 2007;101:1117-23.

14. Rodpai R, Intapan PM, Thanchomnang T, Sanpool O, Janwan P, Laummaunwai $\mathrm{P}$, et al. Strongyloides stercoralis diagnostic polypeptides for human strongyloidiasis and their proteomic analysis. Parasitol Res. 2016;115:4007-12.

15. Rodrigues RM, Sopelete MC, Silva DA, Cunha-Júnior JP, Taketomi EA, Costa-Cruz JM. Strongyloides ratti antigenic components recognized by IgE antibodies in immunoblotting as an additional too for improving the immunodiagnosis in human strongyloidiasis. Mem Inst Oswaldo Cruz. 2004;99:8993. 